\title{
Interface roughness effects on transport in tunnel structures
}

\author{
D. Z.-Y. Ting a) \\ Thomas J. Watson, Sr., Laboratory of Applied Physics, California Institute of Technology, Pasadena, \\ California 91125 and Department of Physics, National Tsing Hua University, Hsinchu, Taiwan 300, \\ Republic of China \\ T. C. McGillb) \\ Thomas J. Watson, Sr., Laboratory of Applied Physics, California Institute of Technology, Pasadena, \\ California 91125
}

(Received 22 January 1996; accepted 16 April 1996)

\begin{abstract}
Direct simulations of interface roughness effects on transport properties of tunnel structures are performed using the planar supercell stack method. The method allows for the inclusion of realistic three-dimensional rough interfacial geometries in transport calculations. For double barrier resonant tunneling structures, we used our method to analyze the effect of roughness at each of the four interfaces, and to test for sensitivity of transport properties to island size and height. Our simulations yields the following conclusions: (1) We find that scattering of off-resonance states into on-resonance states provides the dominant contribution to interface roughness assisted tunneling. Analyses of scattering strength sensitivity to interface layer configurations reveals preferential scattering into $\Delta k_{\|} \approx 2 \pi / \lambda$ states, where $\lambda$ is the island size. (2) We find that roughness at interfaces adjacent to the quantum well can cause lateral localization of wave functions, which increases with island size and depth. Lateral localization can result in the broadening and shifting of transmission resonances, and the introduction of preferential transmission paths. In structures with wide and tall islands, it is possible to find localization over "islands" as well as localization over "oceans." (3) The leading rough interface is the strongest off-resonance scatterer, while rough interfaces adjacent to quantum well are the strongest on-resonance scatterers. The trailing interface is the weakest scatterer. (C) 1996 American Vacuum Society.
\end{abstract}

\section{INTRODUCTION}

While simple one-dimensional models ${ }^{1,2}$ can explain the qualitative behavior of double barrier resonant tunneling diodes, they are less successful at reaching quantitative agreement with experimental results, particularly in predicting peak-to-valley current ratios. The discrepancy has been attributed to electron-phonon scattering, electron-electron interactions, band structure effects, impurity scattering, and interface roughness. In this work, we focus on the effect of interface roughness which, to some extent, can be manipulated by controlling the growth process. Several theoretical works have analyzed interface roughness effects previously. Liu and $\mathrm{Coon}^{3}$ were among the first to approach the subject, modeling rough interfaces with regularly corrugated patterns. Chevoir and Vinter ${ }^{4}$ and Rudberg ${ }^{5}$ studied the topic with Fermi's golden rule. Leo and MacDonald ${ }^{6}$ also treated interface roughness to leading order, but with a technique which preserved unitarity. These perturbative approaches ${ }^{4-6}$ have been successful in dealing with small island sizes. More recently, Johansson ${ }^{7}$ applied the coherent potential approximation which included multiple scattering events. One assumption frequently invoked by many of the previous works is that roughness scattering from the four interfaces in a double barrier structure can be treated independently. Several authors treated interface roughness only at a single interface,

\footnotetext{
${ }^{a}$ Electronic mail: dzt@phys.nthu.edu.tw

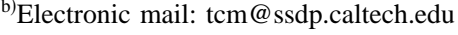

and there is some controversy as to which one of the four interfaces plays the more important role. ${ }^{5,6}$ In this work we formulate the problem in real space with a tight-binding supercell model which allows for arbitrary spatial variations within laterally repeating supercells. Exact solutions are obtained in our calculations. Our model is well-suited for studying effects such as lateral spatial localization, providing a good complement to previous studies.

\section{METHOD}

In the planar supercell stack method (PSSM), we define the active region of a structure as a stack of $N_{z}$ layers perpendicular to the $z$ direction, with each layer containing a periodic array of rectangular planar supercells of $N_{x} \times N_{y}$ sites. A one-band nearest-neighbor tight-binding Hamiltonian is used to describe the potential over this volume of interest. Our model is formally equivalent to the one-band effective mass equation ${ }^{8}$

$$
-\frac{\hbar^{2}}{2} \nabla \cdot \frac{1}{m(\mathbf{x})} \nabla \psi+V(\mathbf{x}) \psi=E \psi,
$$

discretized over a Cartesian grid, and subject to periodic boundary conditions (with supercell periodicity) in the $x$ and $y$ directions, and open boundary conditions in the $z$ direction. Since the planar supercell model may be considered as a multiband model with $N_{x} \times N_{y}$ bands, transmission coefficients for structures described by the planar supercell stack can be determined exactly by the direct application of the multiband quantum transmitting boundary method. ${ }^{9}$ Our 

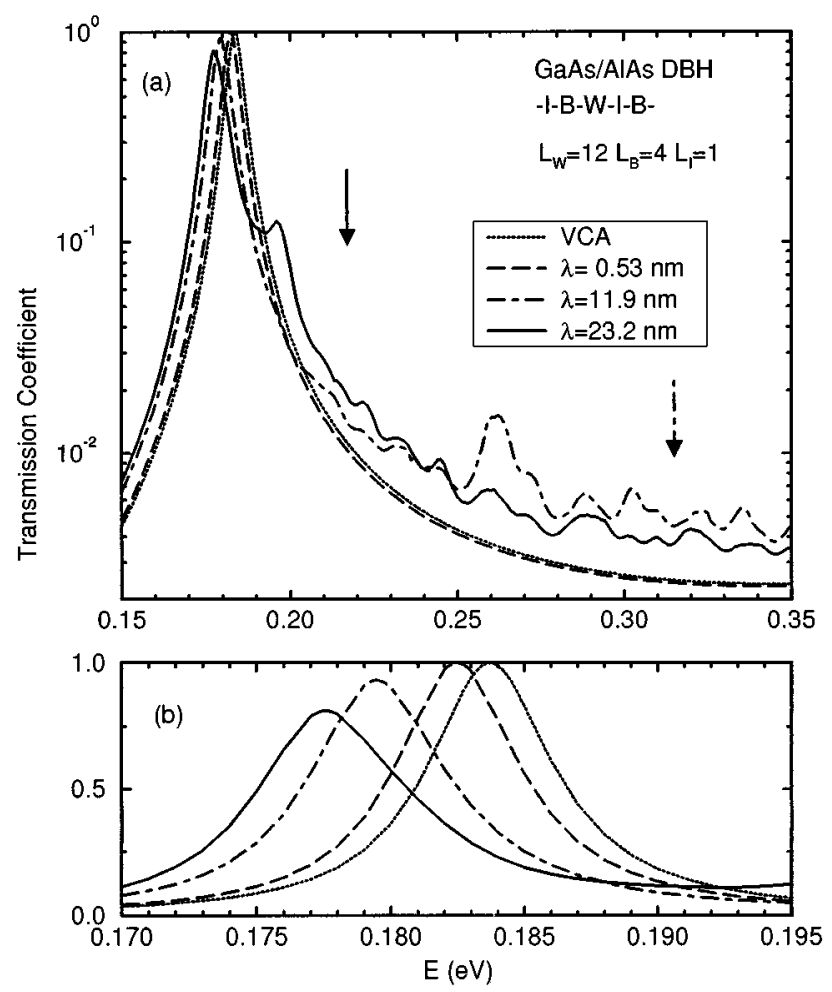

FIG. 1. Transmission coefficients for a set of GaAs/AlAs double barrier structures each containing two rough interfaces (between the leading electrode and the first barrier, and between the quantum well and the second barrier). The different structures have different island sizes, as indicated in the legend. Also included for comparison is a structure where each of the two interface layers is replaced by a $\mathrm{VCA} \mathrm{Al}_{0.5} \mathrm{Ga}_{0.5} \mathrm{As}$ alloy layer. $32 \times 32$ planar supercells are used for this calculation.

method requires accurate and efficient solutions of large sparse linear systems, which is achieved using the quasiminimal residual method. ${ }^{10}$

\section{RESULTS AND DISCUSSION}

We examine how the transmission properties of GaAs/ AlAs double barrier heterostructures are modified by the presence of interface roughness. We consider a set of structures with $L_{W}=12$ (monolayers) GaAs wells and $L_{B}=4$ AlAs barriers. For each GaAs-AlAs interface on the left (incident) side of a AlAs barriers, a 50\% random coverage interface layer is inserted between the pure GaAs and AlAs layers. This configuration corresponds to having rough inverted interfaces and smooth normal interfaces, i.e., if we number the four interfaces 1 through 4 , starting from the incident side, then interfaces 1 and 3 are rough, and interfaces 2 and 4 are smooth. A simulated annealing algorithm ${ }^{11}$ can be used to generate random configurations of interfacial layers with different island sizes. Note that the actual island size, denoted by $\lambda$, depends on the supercell configuration as well as the lateral discretization size. In this article, $32 \times 32$ planar supercells are used in all calculations.

Figure 1 shows transmission spectra near the $n=1$ resonance for the structures discussed above. These structures have average island sizes $(\lambda)$ of $5.3 \AA, 119 \AA$, and $232 \AA$.
For comparison we also show the result for a reference structure in which each of the two rough interfacial layers is replaced by a smooth virtual crystal approximation (VCA) $\mathrm{Al}_{0.5} \mathrm{Ga}_{0.5} \mathrm{As}$ alloy layer. The incident plane waves are chosen to have no in-plane momentum $\left(\mathbf{k}_{\|}=\mathbf{0}\right)$. Our results are similar to those we obtained in a calculation using a smaller $(20 \times 20)$ planar supercell. ${ }^{12} \mathrm{We}$ describe the main points below and refer readers to a more detailed discussion in Reference 12.

Figure 1(a) demonstrates the dependence of interface roughness scattering on island size. Evidently, if the length scale of roughness is small compared to de Broglie wavelength, as in the case of the $\lambda=5.3 \AA$ structure, the interface roughness potential is felt by the electron only in an averaged sense, and thus replacing the rough interfacial layer with a smooth VCA layer is a good approximation. For the structures with larger island sizes of $\lambda=119 \AA$ and $\lambda=232$ $\AA$, the transmission spectra show a series of satellite peaks not found in the reference (VCA) spectrum. The presence of satellite peaks are due to interface roughness assisted resonant tunneling, ${ }^{12}$ which can be pictured as follows: A nominally off-resonance state is elastically scattered by interface roughness into a state with a different $\mathbf{k}_{\|}$. If it so happens that the electron energy with respect to the scattered $\mathbf{k}_{\|}$is on-resonance, then resonant tunneling occurs, leading to enhance transmission. Since coupling between different $\mathbf{k}_{\|}$ states is determined by the interface roughness configuration, we would expect to find preferential scattering into $\Delta k_{\|} \approx 2 \pi / \lambda$ states. $^{12}$ Arrows in Figure 1(a) indicates positions where preferential scattering is expected to occur for the $\lambda=119 \AA$ and $\lambda=232 \AA$ structures based on the simple estimate using island sizes; the results seem to confirm our expectations.

In principle, interface roughness scattering can result in continuous range of $\Delta \mathbf{k}_{\|}$. In our model, an incident wave can only be scattered into states with $\Delta \mathbf{k}_{\|}$equal to a reciprocal lattice vector $\mathbf{g}$ of the (finite) planar supercell. Thus, the appearance of discrete satellite peaks in our calculate transmission spectra is an artifact of the finite supercell size effect. This artifact can be mitigated by the use of larger supercells. The results of the present $32 \times 32$ supercell calculation shows closer spacing between satellite peaks than the previous $20 \times 20$ supercell calculation. ${ }^{12}$ In the limit of very large supercell sizes, the spacings between the satellite peaks would become smaller than the resonance peak width, and the satellite peaks would then coalesce, giving a much smoother appearance.

Figure 1(b) shows that the main resonance peak broadens and shifts to lower energy as island size increases. We attribute this to lateral wave function localization. The rough interfacial layer between the quantum well and the second barrier introduces well width fluctuation. If the island sizes are sufficiently large, we could consider the quantum well as consisting of wide-well $\left(L_{W}=13\right.$, in this example) and narrow-well $\left(L_{W}=12\right)$ regions. If a quasibound level is localized (laterally) in the wide-well region, its resonance energy would be lowered. Evidently, the degree of lateral lo- 

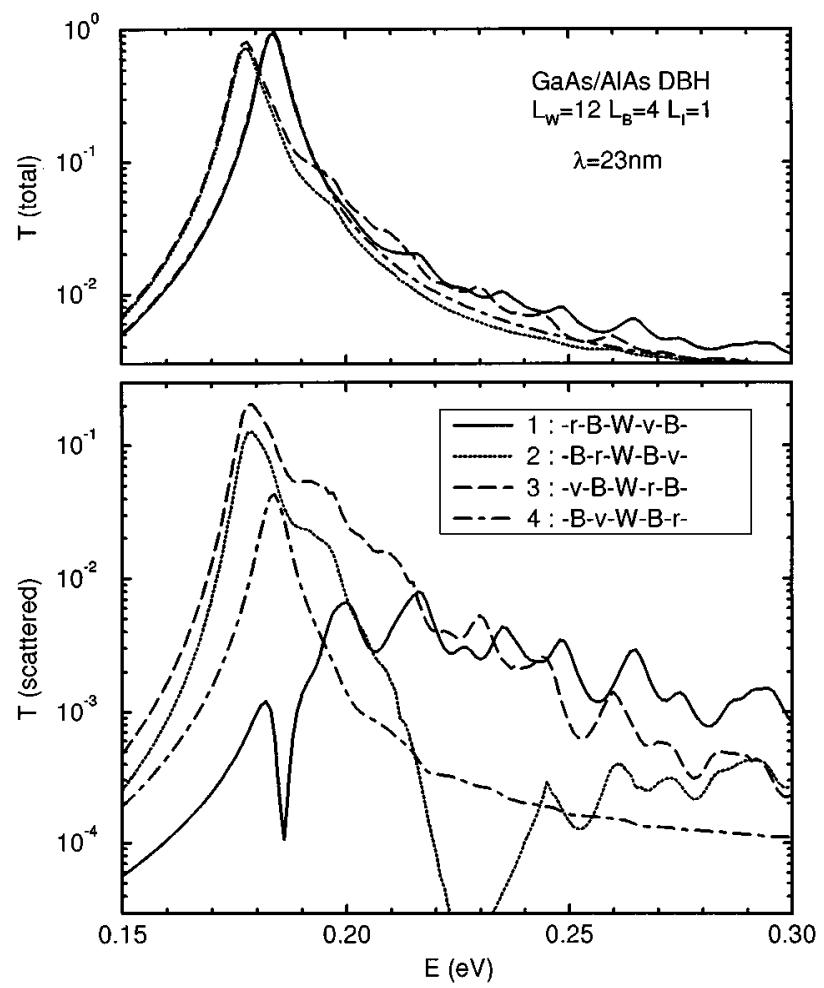

Fig. 2. Transmission coefficients for a set of GaAs/AlAs double barrier structures with interface roughness only in one of the four interfacial layers. The total transmission coefficient is shown in the top panel, while the transmission coefficient for only the scattered components (i.e., $\mathbf{k}_{\|}^{\text {incident }} \neq \mathbf{k}_{\|}^{\text {transmitted }}$ ) is shown in the bottom panel.

calization increases with island size. Reference 12 examines wave function localization directly; it also demonstrates preferential transmission paths resulting from wave function localization. Note also that since the second barrier is thinner where the quantum well is wider, an electron localized in the wide-well/thin-barrier region can escape with more ease through the thinner barriers, leading to shorter quasibound state lifetime, or broader resonance, as seen in Figure 1(b).

In Figure 2 we explore the role of the individual interfaces. Strictly speaking, with wave function coherence across the entire device structure, encompassing all four interfaces, we cannot separate out the effects due to the different rough interfacial layers. Nevertheless, it is instructive to study the following. We examine four different configurations. Configuration 1 is similar to the configurations discussed earlier, except that the third interface layer (between the quantum well and the second barrier) is now replaced by a smooth VCA layer, leaving only the leading interface (1) rough. We use "-r-B-W-v-B-" to denote the layer sequence of this structure. Configurations 2,3 , and 4 are similarly constructed, each with one rough interface and one VCA interface; their layer sequences are shown in the inset of Figure 2. We note that the main resonance peak is down-shifted only in configurations 2 and 3, since lateral localization of wave function only occurs if one of the interfaces adjacent to the quantum well is rough. We also note that interface roughness

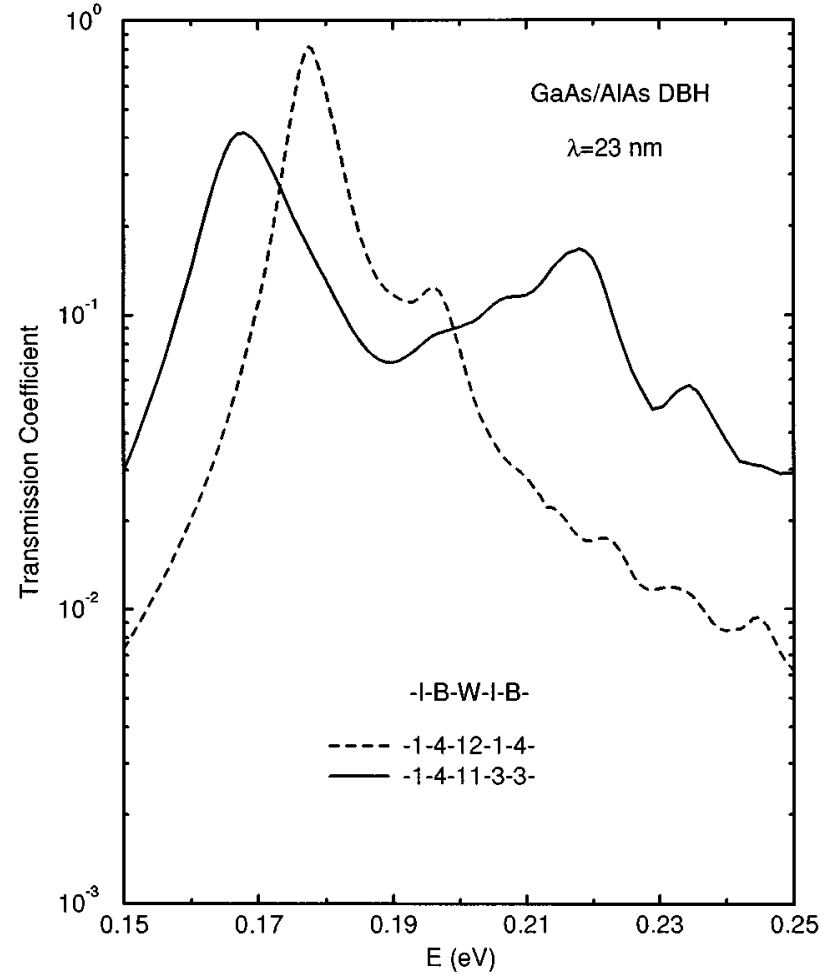

FIG. 3. Transmission coefficients for two GaAs/AlAs double barrier structures. The rough interfacial layer between the quantum well and the barrier for one structure (dashed line) is one monolayer thick, and for the other (solid line) is three monolayers.

scattering is the least important in configuration 4 , where the rough interfacial layer is located away from the incident side. This is because unless the incident electron is on-resonance, and the transmission coefficient is reasonably large, the wave function is largely located on the incident side, with extremely small probability of penetrating across the device structure to the transmitted side to sense the roughness at the far interface. The same argument explains the fact that offresonance scattering is the largest in configuration 1, where the leading interface is rough.

Figure 3 shows the transmission spectra of two structures with similar island sizes but different island heights. The first structure is identical to the $\lambda=232 \AA$ structure from Figure 1. The second structure is similar to the first one, except that the random-alloy interfacial region between the quantum well and the second barrier is now expanded into three monolayers (at the expense of the surrounding well and barrier layers, so as to keep the effective well width the same). We note that the main resonance peak of the second structure (with taller islands) is lower than the first structure; its wave function also shows a higher degree of lateral confinement over the wide-well region. It is also interesting to note that the wave function for the transmission peak at $218 \mathrm{meV}$ shows the opposite type of lateral confinement, i.e., the wave functions is localized over the islands themselves (the narrow-well regions) rather than over the "ocean." This indicates a splitting of the $n=1$ quasibound state into orthogo- 
nal states laterally localized in different regions of the quantum well.

\section{SUMMARY}

We studied the effect of interface roughness on resonant tunneling in double barrier structures using a tight-binding supercell model. We find that interface roughness assisted scattering of off-resonance states into on-resonance state provides the dominant scattering channel. We showed that scattering strength is sensitive to the configuration of the roughness layer, and in particular, we find preferential scattering into $k_{\|} \approx 2 \pi / \lambda$ states $(\lambda=$ island size). We showed that lateral wave function localization increases with island size and depth, and is responsible for broadening and shifting of transmission resonances. Both types of lateral localization (over "islands" or over "ocean") are possible. We also explored the roles of the individual interfaces, and showed that the leading rough interface is the strongest off-resonance scatterer, while the rough interfaces adjacent to quantum well are the strongest on-resonance scatterers.

\section{ACKNOWLEDGMENTS}

This work was supported by the Office of Naval Research (ONR) under Grant No. N00014-92-J-1845. One of the authors (D.Z.T.) acknowledges partial support from Department of Physics, National Tsing Hua University (Hsinchu).

${ }^{1}$ R. Tsu and L. Esaki, Appl. Phys. Lett. 22, 562 (1973).

${ }^{2}$ B. Ricco and M. Ya. Azbel, Phys. Rev. B 29, 1970 (1983).

${ }^{3}$ H. C. Liu and D. D. Coon, J. Appl. Phys. 64, 6785 (1988).

${ }^{4}$ F. Chevoir and B. Vinter, Appl. Phys. Lett. 55, 1859 (1989); Phys. Rev. B 47, 7260 (1993).

${ }^{5}$ B. G. R. Rudberg, Semicond. Sci. Technol. 5, 600 (1990).

${ }^{6}$ J. Leo and A. H. MacDonald, Phys. Rev. Lett. 64, 817 (1990); Phys. Rev. B 43, 9763 (1991).

${ }^{7}$ P. Johansson, Phys. Rev. B 46, 12865 (1992).

${ }^{8}$ D. J. BenDaniel and C. B. Duke, Phys. Rev. 152, 683 (1966).

${ }^{9}$ D. Z.-Y. Ting, E. T. Yu, and T. C. McGill, Phys. Rev. B 45, 3583 (1992).

${ }^{10}$ R. W. Freund and N. M. Nachtigal, Numer. Math. 60, 315 (1991); RIACS, NASA Ames Research Center Technical Report 90.51, Dec. 1989.

${ }^{11}$ N. Metropolis, A. Rosenbluth, M. Rosenbluth, A. Teller, and E. Teller, J. Chem. Phys. 21, 1087 (1953).

${ }^{12}$ D. Z.-Y. Ting, S. K. Kirby, and T. C. McGill, Appl. Phys. Lett. 64, 2004 (1994). 